\title{
Young Women with Breast Cancer: Chemotherapy or Surgery First? An Evaluation of Time to Treatment for Invasive Breast Cancer
}

\author{
Evelyne Guay, BSc ${ }^{1}$ (D), Erin Cordeiro, MD, MSc, FRCSC ${ }^{2}$, and Amanda Roberts, MD, MPH, FRCSC ${ }^{3,4}$ \\ ${ }^{1}$ School of Medicine, University of Ottawa, Ottawa, ON, Canada; ${ }^{2}$ Department of Surgery, University of Ottawa, Ottawa, \\ ON, Canada; ${ }^{3}$ Department of Surgery, University of Toronto, Toronto, ON, Canada; ${ }^{4}$ Sunnybrook Health Sciences Centre, \\ Toronto, ON, Canada
}

\begin{abstract}
Background. Breast cancer in young women (ages 18-40 years) is rare, yet remains a leading cause of cancer-related death. Time to treatment (TTT) is an increasingly important factor in breast cancer outcomes, specifically time to systemic therapy. Our objective was to review patterns of care for young women presenting with invasive breast cancer and compare TTT for surgery first versus neoadjuvant chemotherapy (NAC).

Patients and Methods. A retrospective chart review of young women with non-metastatic, non-inflammatory invasive breast cancer between 2012 and 2018 at a single institution was completed. The primary outcome was time to first treatment (surgery or NAC).

Results. One hundred forty-two young women were treated for invasive breast cancer during the study period. The majority of patients underwent surgery first $(57.7 \%)$ compared with NAC (42.3\%). Women who underwent NAC were more likely to have abnormal lymph nodes on imaging $(p=0.002)$ and clinical exam $(p<0.0001)$ and were also more likely to have larger tumor sizes $(p<0.05)$. The majority of triple negative patients underwent NAC first (88\% [14/16]). Median TTT was significantly longer for surgery (27 [range 7-70] days)
\end{abstract}

Erin Cordeiro and Amanda Roberts are co-senior authors.

(C) Society of Surgical Oncology 2021

First Received: 1 July 2021

Accepted: 3 November 2021;

Published Online: 26 November 2021

A. Roberts, MD, MPH, FRCSC

e-mail: amanda.roberts@sunnybrook.ca versus (20.5 [3-50] days) chemotherapy $(p=0.004)$. Median number of additional hospital visits prior to surgery was 4 (range 1-8) versus $5(0-11)$ for NAC $(p<0.001)$.

Conclusions. Young women with breast cancer who undergo NAC have a shorter TTT and clinically similar median number of hospital visits compared with women undergoing surgery first. These results support the use of NAC in young women, when indicated, as additional workup and consultations prior to NAC do not delay care.

Breast cancer is the most common cancer diagnosis in women, being prevalent in both older and young women. ${ }^{1}$ However, the disease differs considerably between the two cohorts. Compared with older women, breast tumors in young women are likely to be larger in size, higher grade, node positive, and have a higher number of nodes involved. ${ }^{2}$ Breast cancer in young women has been regarded as a "unique biological entity." ${ }^{3}$ A landmark retrospective study of nearly 500,000 women with invasive breast cancer demonstrated that breast cancer in young women has distinct oncologic signaling pathways and chemosensitivities, a finding which may have important therapeutic implications. ${ }^{4}$ Young women also have a higher incidence of triple negative cancers compared with older women. ${ }^{5}$ Triple-negative tumors have a worse prognosis compared with tumors that have at least one positive receptor. ${ }^{6,7}$ This worse prognosis may be a result of less targeted therapies available for triple negative carcinomas compared with estrogen receptor positive and HER2 positive cancers. Young women are also more likely to present with a self-detected cancer, and more likely to be diagnosed at a later stage. ${ }^{8}$ Higher breast density in young 
woman is difficult to interpret on mammography, making diagnostic imaging also more challenging in this cohort. ${ }^{8}$ Sadly, young women are up to 1.5 times more likely to die from their breast cancer compared with older women, ${ }^{9,10}$ and more likely to have local recurrence. ${ }^{11,12}$ Therefore, young age in and of itself is regarded as a risk factor for recurrence. ${ }^{13}$ Interestingly, young women are more likely to require chemotherapy in their treatment course yet traditional indications for neoadjuvant chemotherapy (NAC) are often applied to young women. These practices persist despite trends in the literature towards a survival advantage to NAC for specific cancer subtypes in this patient cohort. ${ }^{14}$ Specifically, age is not a factor in selecting chemotherapeutic regimens to treat breast cancer. ${ }^{13}$ However, older women are also more likely to have multiple comorbidities, which are taken into consideration when making treatment decisions. Other factors, including hormone and HER2 receptor status, and tumor grade, proliferation, and stage, should also be taken into account when considering therapeutic regimens. ${ }^{15}$ Older women, specifically patients over the age of 65 years, are more likely to require dose modifications in systemic therapies. ${ }^{16,17}$

Time to treatment (TTT) is an increasingly important factor in breast cancer outcomes and has gained recent attention in the literature. TTT is defined as the period from diagnosis of breast cancer, or alternatively from initial consultation to receipt of first breast cancer treatment. TTT may also be assessed in terms of time to receipt of adjuvant therapy, for example, time to adjuvant chemotherapy following breast cancer surgery. Specifically, longer time to breast cancer surgery following diagnosis is associated with decreased overall survival (OS), breast-cancer mortality, and overall mortality. ${ }^{18,19}$ Longer time to surgery following NAC is also associated with worse OS and disease-free survival (DFS) ${ }^{20,21}$ Furthermore, longer time to adjuvant chemotherapy and radiation therapy are associated with worse OS and DFS. ${ }^{22-25}$

To our knowledge, there is limited evidence surrounding time to NAC in young women. Therefore, the objective of this study was to review the patterns of care for women age 40 years or younger presenting with invasive breast cancer and specifically compare the TTT for surgery versus NAC. Number of additional visits to the hospital following cancer diagnosis prior to first treatment received was also evaluated.

\section{PATIENTS AND METHODS}

A single-institution retrospective chart review was conducted on all women diagnosed with non-metastatic invasive breast cancer between the ages of 18 and 40 years who were referred to a regional breast cancer center. Records from 1 January 2012 to 31 December 2018 were searched for patients with a primary diagnosis of nonmetastatic invasive breast cancer who received either surgery or NAC as their first treatment. All patients underwent surgery during the study period. Patients with a diagnosis of ductal carcinoma in situ, inflammatory breast cancer, or recurrent breast cancer were excluded. Patients who did not undergo surgery or NAC during the study period were excluded. Patients whose workup and care were not entirely at the center of interest were excluded as the number of additional hospital visits could not be assessed in these patients.

Data was collected regarding patient, clinical, and tumor characteristics; additional consultations and diagnostic imaging; and dates of first surgery or systemic therapy. Race, ethnicity, and socioeconomic data was not reported as this information was not available. TTT was recorded as number of days between initial consultation with a breast cancer specialist (surgical, medical, or radiation oncologist) to the date of receipt of first treatment, either surgery or NAC. Visits with breast cancer specialists at the single institution of interest were not coordinated to occur on the same day. Number of additional hospital visits was recorded as the number of distinct dates the patient visited the hospital between initial consultation and first treatment. For example, an ECHO and port insertion that occurred on the same date was recorded as a single hospital visit. Initial consultation and first treatment were not counted as additional visits. As this project is a quality improvement initiative, it was exempt from research ethics approval.

Univariate analysis was performed to compare characteristics of patients who were treated with surgery first to those who were treated with NAC first. Chi-square test (or Fisher's exact test in cases where expected cell count was $<5$ ) was used to compare categorical variables. A two-sample $t$-test or Wilcoxon rank sum test was used to examine continuous variables (normally and non-normally distributed, respectively). A multivariable log transformed linear regression was performed to identify independent predictors of TTT. Predictors included were determined by clinical significance a priori. All statistical analyses were performed using SAS 9.3 (SAS Institute Inc., Cary, NC, USA).

\section{RESULTS}

Between 1 January 2012 and 31 December 2018, 142 young women $(<40$ years) underwent treatment for invasive breast cancer at our regional cancer center. Of these women, $82(58 \%)$ underwent surgery first, while 60 (42\%) underwent NAC as their first treatment. Women who 
underwent NAC first were more likely to have abnormal lymph nodes on imaging $(p=0.002)$ and clinical exam $(p<0.0001)$, and more likely to have larger tumor sizes $(p<0.05)$. The majority of triple negative and HER $2+\mathrm{ve}$ patients underwent NAC first (88\% [14/16] and 96\% [17/ 18] respectively). Additional clinical and imaging characteristics of the patient cohorts are presented in Table 1.

The median TTT for women undergoing surgery first was 27 days (range 7-70 days). The median TTT for women undergoing NAC first was 20.5 days (range 3-50 days). The difference in TTT between these two cohorts was statistically significant $(p=0.007)$. The median number of days visiting the hospital prior to the first treatment for women receiving surgery first was 4 (range 1-8). The median number of days visiting the hospital prior to the first treatment for women receiving NAC first was 5 (range $0-11)$. The difference in number of days attending the hospital between these two cohorts was statistically significant $(p<0.0001)$. Reasons for additional visits to the hospital were for diagnostic imaging, biopsies, and visits with allied health such as social workers or physiotherapists.

On multivariable regression, patients undergoing NAC compared with surgery as first treatment, had a $21.4 \%$ shorter TTT $(p=0.035)$ - the equivalent of approximately 5 days. Tumor size and presence of abnormal lymph nodes on preoperative imaging were not independently associated with a reduced TTT.

\section{DISCUSSION}

Young women with breast cancer who undergo NAC as their first treatment have a significantly shorter TTT despite more advanced disease compared with those undergoing surgery first. While this difference is statistically significant, further research is required to assess whether it has an effect on survival or oncologic outcomes. Young women receiving NAC had a greater incidence of positive axillary lymph nodes and larger tumor sizes compared with the surgery-first cohort. This finding of more advanced disease in NAC patients is likely explained by recognizing these clinical characteristics as relative indications for NAC. ${ }^{26}$ Patients undergoing NAC had, on average, one more hospital visit than patients receiving surgery first.

We sought to expand TTT literature on breast cancer specifically in young women given the unique features of this population highlighted in the introduction and the paucity of TTT data in this cohort. To our knowledge, this is the first study to compare TTT for surgery versus NAC specifically in young women with breast cancer. Akhtar et al. compared time to NAC versus surgery in a broader age range of patients with invasive breast cancer, aged 18 years or older, and found similar results to our younger cohort. In their study, median time to NAC was 22 days (similar to our median of 20.5 days) and median time to surgery was 31 days (our median was 27 days). ${ }^{27}$ TTT was similar between the aforementioned study and our study, despite the fact that these analyses assessed different models of healthcare-the American and Canadian healthcare systems.

While survival outcomes regarding delays in TTT for breast cancer surgery and other adjuvant therapies have been assessed in the literature, associations between time to $N A C$ and survival is not well studied. Livingston-Rosanoff et al. assessed time to NAC in a large retrospective review of women aged 18 years or older diagnosed with stage I to III invasive breast cancer and found a median time to NAC of 4 weeks. In their analysis, time to NAC was not associated worse survival outcomes in triple negative or HER2 + cancers (hormone receptor positive and HER2patients were excluded from the study), with authors concluding that delays of initiation of NAC up to 6 months do not impact survival outcomes. However, this study had a broader age range than our study, with a median age of 52 years of age, which is significantly older than our median age of 35.8. Further studies would need to assess correlations between time to NAC and OS specifically in young women, given that other studies have shown that NAC may offer a survival benefit and higher likelihood of pathologic complete response (pCR) in young women. ${ }^{14,26}$ Conversely, associations between time to surgery and OS have been better established. Longer time to surgery following breast cancer diagnosis is associated with decreased OS, and increased breast-cancer mortality and overall mortality. ${ }^{18,19}$ However, delays less than 30 days are unlikely to be statistically significant. As such, when indicated, NAC should be considered as initial form of treatment in young women with invasive breast cancer.

Several studies have found that the use of NAC in the treatment of locally advanced breast cancer has been increasing over time. ${ }^{26,28,29}$ The indications for NAC include node positive or T3 tumors as well as tumors at least $2 \mathrm{~cm}$ in size that are triple negative or HER2.$+{ }^{30,31}$ Advantages of NAC include downstaging the primary tumor to improve operability and potentially enable breastconserving surgery in patients previously requiring mastectomy; allowing for earlier treatment of distant micrometastases; enabling assessment of tumor response to targeted agents; enabling prognostication given clinical and pathologic response; and decreasing the necessity for axillary lymph node dissection in node-positive patients. $^{32,33}$ The potential for breast conservation in a young population should not be overlooked for several reasons including body image, sexual health, and anxiety issues associated with more extensive surgery. ${ }^{34}$ More 
TABLE 1 Baseline characteristics of breast cancer patients undergoing neoadjuvant chemotherapy

\begin{tabular}{|c|c|c|c|c|}
\hline & $\begin{array}{l}\text { Entire cohort } \\
n=142(\%)\end{array}$ & $\begin{array}{l}\text { Surgery first } \\
n=82(\%)\end{array}$ & $\begin{array}{l}\text { NAC } \\
n=60(\%)\end{array}$ & $p$ value \\
\hline Age, years: mean [SD] & $35.8[4.2]$ & $36.7[4.1]$ & $34.6[4.1]$ & $<0.001$ \\
\hline Primary diagnosis: $n(\%)$ & & & & 0.25 \\
\hline IDC & $130(91.6)$ & $73(89.0)$ & $57(95.0)$ & \\
\hline ILC & $5(3.5)$ & $4(4.9)$ & $1(1.7)$ & \\
\hline Invasive mixed carcinoma & $1(0.7)$ & 0 & $1(1.7)$ & \\
\hline Other & $6(4.2)$ & $5(6.1)$ & $1(1.7)$ & \\
\hline Receptors on biopsy: $n(\%)$ & & & & 0.002 \\
\hline ER+PR+HER-2- & $40(46.0)$ & $23(79.3)$ & $17(29.3)$ & \\
\hline ER+PR+HER-2+ & $11(12.6)$ & $1(3.4)$ & $10(17.2)$ & \\
\hline ER-PR-HER-2+ & $5(5.8)$ & 0 & $5(8.6)$ & \\
\hline ER-PR-HER-2- & $16(18.4)$ & $2(6.9)$ & $14(24.1)$ & \\
\hline ER+PR-HER-2- & $10(11.5)$ & $2(6.9)$ & $8(13.8)$ & \\
\hline ER+PR-HER-2+ & $2(2.3)$ & 0 & $2(3.4)$ & \\
\hline Missing & 55 & & & \\
\hline Receptors on final surgical excision: $n(\%)$ & & & & $<0.0001$ \\
\hline ER+PR+HER-2- & $33(23.6)$ & $30(37.0)$ & $3(5.1)$ & \\
\hline ER+PR+HER-2+ & $10(7.1)$ & $9(11.1)$ & $1(1.7)$ & \\
\hline ER-PR-HER-2+ & $5(3.6)$ & $4(4.9)$ & $1(1.7)$ & \\
\hline ER-PR-HER-2- & $9(6.4)$ & $5(6.2)$ & $4(6.8)$ & \\
\hline ER+PR-HER-2- & $9(6.4)$ & $4(4.9)$ & $5(8.5)$ & \\
\hline ER+PR-HER-2+ & $2(1.4)$ & $1(1.2)$ & $1(1.7)$ & \\
\hline Not repeated & $25(17.9)$ & $1(1.2)$ & $24(40.7)$ & \\
\hline Other & $4(2.9)$ & $4(4.9)$ & 0 & \\
\hline Missing & 2 & & & \\
\hline Breast density $n(\%)$ & & & & 0.63 \\
\hline Extremely dense & $22(15.6)$ & $12(14.6)$ & $10(17.0)$ & \\
\hline Heterogeneous & $63(44.7)$ & $34(41.5)$ & $29(49.2)$ & \\
\hline Scattered & $20(14.2)$ & $13(15.8)$ & $7(11.9)$ & \\
\hline Fatty & $2(1.4)$ & $2(2.44)$ & 0 & \\
\hline Not reported & $34(24.11)$ & $21(25.6)$ & $13(22.0)$ & \\
\hline Missing & 1 & & & \\
\hline BI-RADS at time of $\mathrm{Bx} n(\%)$ & & & & 0.5 \\
\hline $4 \mathrm{~A}$ & $17(12.7)$ & $11(13.9)$ & $6(10.9)$ & \\
\hline 4B & $26(19.4)$ & $19(24.0)$ & $7(12.7)$ & \\
\hline $4 \mathrm{C}$ & $2(1.5)$ & $1(1.3)$ & $1(1.8)$ & \\
\hline 4 & $2(1.5)$ & $1(1.3)$ & $1(1.8)$ & \\
\hline 5 & $87(64.9)$ & $47(59.5)$ & $40(72.7)$ & \\
\hline Missing & 8 & & & \\
\hline \multicolumn{5}{|c|}{ Breast imaging performed prior to consultation: $n(\%)$} \\
\hline Mammogram & $131(92.2)$ & $76(92.7)$ & $55(91.7)$ & 0.83 \\
\hline Breast ultrasound & $142(100.0)$ & $82(100.0)$ & $60(100.0)$ & $\mathrm{n} / \mathrm{a}$ \\
\hline Axillary ultrasound & $127(89.4)$ & $68(82.9)$ & $59(98.3)$ & 0.003 \\
\hline Breast MRI & $47(33.1)$ & $31(37.8)$ & $16(26.7)$ & 0.164 \\
\hline Breast biopsy(ies) & $139(97.9)$ & $82(100.0)$ & $57(95.0)$ & 0.041 \\
\hline ALN Bx & $43(30.3)$ & $17(20.7)$ & $26(43.3)$ & 0.004 \\
\hline Abnormal LNs on preconsult imaging & $66(46.8)$ & $28(34.2)$ & $38(64.4)$ & 0.002 \\
\hline Palpable LNs on clinical exam & $42(29.6)$ & $12(14.6)$ & $30(50.0)$ & $<0.0001$ \\
\hline
\end{tabular}


Table 1 (continued)

\begin{tabular}{|c|c|c|c|c|}
\hline & $\begin{array}{l}\text { Entire cohort } \\
n=142(\%)\end{array}$ & $\begin{array}{l}\text { Surgery first } \\
n=82(\%)\end{array}$ & $\begin{array}{l}\text { NAC } \\
n=60(\%)\end{array}$ & $p$ value \\
\hline Size reported on preconsult imaging & $129(91.5)$ & $75(91.5)$ & $54(91.5)$ & 0.99 \\
\hline Median size of breast mass [range] $(\mathrm{cm})$ & $2.6[0.5-9.8]$ & $2.2[0.5-9.0]$ & $3.6[0.9-9.8]$ & $<0.0001$ \\
\hline Multifocal disease suspected & $39(27.7)$ & $22(26.8)$ & $17(28.8)$ & 0.2 \\
\hline Multicentric disease suspected & $27(19.2)$ & $12(14.6)$ & $15(25.4)$ & \\
\hline Suspicion of inflammatory on exam & $3(2.1)$ & 0 & $3(5.0)$ & 0.041 \\
\hline $\mathrm{Bx}$ results available at date of consultation & $135(95.1)$ & 77 (93.9) & $58(96.7)$ & 0.45 \\
\hline
\end{tabular}

$I D C$ invasive ductal carcinoma, $I L C$ invasive lobular carcinoma, ER estrogen receptor, $P R$ progesterone receptor, HER2 human epidermal growth factor receptor 2, BI-RADS Breast Imaging Reporting and Database System score

recently, during the COVID-19 pandemic, utilization of NAC has increased due to deferred breast cancer surgeries as a result of operating room closures. ${ }^{35}$ With the uncertainty posed by future waves of COVID-19 and emergence of new virus variants, it is critical to consider the viability of NAC as a first treatment option in young women when surgery may be delayed.

Patients receiving NAC first had one additional visit prior to initiating treatment compared with those undergoing surgery first, with a median of five visits for NAC patients compared to four for surgery first. It is important to consider the burden of repeat hospital visits on the patient. Each additional visit may have financial implications: time off work, commuting and parking fees, childcare fees especially in women less than 40 years with young children; as well as psychosocial implications, such as anxiety or fatigue with visiting the hospital. Every visit to the hospital may be a reminder to the patient of their disease. As such, it is beneficial to consider implementation of multidisciplinary care clinics in the treatment of young women with breast cancer to reduce the financial and emotional burden for patients associated with repeat hospital visits. Young women in particular may benefit from a multidisciplinary approach to treatment given unique fertility, sexuality, and pregnancy considerations in this cohort. $^{13}$

Our study was limited by the retrospective nature of the data as there may be missing entries for clinical variables, such as tumor receptors, or hospital visits. Some patients may have completed staging investigations at different hospital networks or completed staging investigations prior to the initial consult visit. While we attempted to determine and exclude these patients, this was limited by the retrospective nature of the data. As such, the median number of hospital visits may have been underestimated. We were also unable to ascertain the reason for possible delays in treatment based on our data. We recognize that personal patient factors and demographics may impact TTT. For instance, race and ethnicity has been demonstrated in the literature to be associated with delays in treatment. ${ }^{36}$ Unfortunately, race and ethnicity data were not available for our analysis; this presents an interesting future study to assess associations between TTT and race and ethnicity, comparing NAC versus surgery cohorts. Furthermore, preservation of fertility is a commonly cited reason for treatment delay. ${ }^{13}$ Treatment delays in pursuit of fertility preservation are more likely to occur in patients receiving surgery first, whereas patients undergoing NAC are more likely to decline fertility preservation in order to pursue timely treatment. ${ }^{37}$ This presents a potential source of bias towards treatment delays in the surgery-first cohort. Furthermore, confounding bias (both measured and unmeasured) exists between the two cohorts, including the fact that the NAC cohort was more likely to have node positive disease, larger tumor sizes, and triple negative tumors as compared with the surgery-first cohort.

In addition, TTT is often regarded in the literature as time from initial diagnosis to first treatment, though some define TTT beginning from initial consultation. As the primary goal of our quality initiative is to implement a same-day multidisciplinary clinic, we chose to evaluate the TTT from the first consultation to first treatment as this is the portion of care that our proposed clinic will impact. In addition, due to limitations of our dataset, the date of diagnosis was not always reliable.

Encouragingly, TTT for both surgery and NAC is less than 30 days within our single-payer healthcare organization. This timeframe meets provincial guidelines of 28 days for time to surgery for non-immediately life-threatening cancers ${ }^{38}$ however, no guidelines exist for time to NAC. A Canadian National Expert consensus recognized that there are gaps in the literature regarding NAC, especially when compared with other forms of breast cancer treatment. ${ }^{39}$ In light of the paucity of evidence, they suggest that breast cancer providers shoulder consider consensus statements that takes high-level evidence into account as 
the best approach to considering NAC in a patient's breast cancer treatment pathway. Our study adds to the evidence surrounding NAC, particularly in young women, which experts can consider when making treatment decisions.

In summary, the results of this study support the use of neoadjuvant chemotherapy in young women with breast cancer, when indicated, to ensure timely access to care. Time to NAC can be significantly shorter than time to surgery, by 6.5 days in the current study, which may have a psychological impact on young women. Further assessment is required to assess whether this amount of time is clinically significant in regard to survival and psychological outcomes in young women.

\section{FUNDING None.}

\section{REFERENCES}

1. Bleyer A, Barr R, Hayes-Lattin B, et al. The distinctive biology of cancer in adolescents and young adults. Nat Rev Cancer. 2008;8(4):288-98. https://doi.org/10.1038/nrc2349.

2. Plichta JK, Thomas SM, Vernon R, et al. Breast cancer tumor histopathology, stage at presentation, and treatment in the extremes of age. Breast Cancer Res Treat. 2020;180:227-35. h ttps://doi.org/10.1007/s10549-020-05542-4.

3. Anders CK, Johnson R, Litton J, Phillips M, Bleyer A, Author SO. Breast cancer before age 40 years NIH public access author manuscript. Semin Oncol. 2009;36(3):237-49. https://doi.org/10. 1053/j.seminoncol.2009.03.001.

4. Anderson WF, Bingshu AE, Chen E, Ae LAB, Devesa SS. Qualitative age interactions (or effect modification) suggest different cancer pathways for early-onset and late-onset breast cancers. Cancer Causes Control. 2007. https://doi.org/10.1007/ s10552-007-9057-x.

5. Tichy JR, Lim E, Anders CK. Breast cancer in adolescents and young adults: a review with a focus on biology. J Natl Compr Cancer Netw. 2013;11(9):1060-9. https://doi.org/10.6004/jnccn. 2013.0128.

6. Cleator S, Heller W, Coombes RC. Triple-negative breast cancer: therapeutic options. Lancet Oncol. 2007;8(3):235-44. https://doi. org/10.1016/S1470-2045(07)70074-8.

7. Assi HA, Khoury KE, Dbouk H, Khalil LE, Mouhieddine TH, El Saghir NS. Epidemiology and prognosis of breast cancer in young women. J Thorac Dis. 2013;5 Suppl 1(Suppl 1):S2-8. h ttps://doi.org/10.3978/j.issn.2072-1439.2013.05.24.

8. Foxcroft LM, Evans EB, Porter AJ. The diagnosis of breast cancer in women younger than 40. Breast. 2004;13(4):297-306. h ttps://doi.org/10.1016/j.breast.2004.02.012.

9. Bharat A, Aft RL, Gao F, Margenthaler JA. Patient and tumor characteristics associated with increased mortality in young women ( $\leq 40$ years) with breast cancer. $J$ Surg Oncol. 2009;100(3):248-51. https://doi.org/10.1002/jso.21268.

10. Gnerlich JL, Deshpande AD, Jeffe DB, Sweet A, White N, Margenthaler JA. Elevated breast cancer mortality in women younger than age 40 years compared with older women is attributed to poorer survival in early-stage disease. J Am Coll Surg. 2009;208(3):341-7. https://doi.org/10.1016/j.jamcollsurg.2 008.12.001.

11. De Bock GH, Van Der Hage JA, Putter H, Bonnema J, Bartelink $\mathrm{H}$, Van De Velde CJ. Isolated loco-regional recurrence of breast cancer is more common in young patients and following breast conserving therapy: long-term results of European Organisation for Research and Treatment of Cancer studies. Eur J Cancer. 2006;42(3):351-6. https://doi.org/10.1016/j.ejca.2005.10.006.

12. Wapnir IL, Anderson SJ, Mamounas EP, et al. Prognosis after ipsilateral breast tumor recurrence and locoregional recurrences in five national surgical adjuvant breast and bowel project nodepositive adjuvant breast cancer trials. $J$ Clin Oncol. 2006;24(13):2028-37. https://doi.org/10.1200/JCO.2005.04. 3273.

13. Reyna C, Lee MC. Breast cancer in young women: special considerations in multidisciplinary care. J Multidiscip Healthc. 2014. https://doi.org/10.2147/JMDH.S49994.

14. Loibl S, Jackisch C, Lederer B, et al. Outcome after neoadjuvant chemotherapy in young breast cancer patients: a pooled analysis of individual patient data from eight prospectively randomized controlled trials. Breast Cancer Res Treat. 2015;152(2):377-87. h ttps://doi.org/10.1007/s10549-015-3479-z.

15. Suter MB, Pagani O. Should age impact breast cancer management in young women? Fine tuning of treatment guidelines. Ther Adv Med Oncol. 2018;10:1758835918776923. https://doi.org/10. $1177 / 1758835918776923$.

16. Raza S, Welch S, Younus J. Relative dose intensity delivered to patients with early breast cancer: Canadian experience. Curr Oncol. 2009;16(6):8-12. https://doi.org/10.3747/CO.V16I6.311.

17. Shayne M, Crawford J, Dale DC, Culakova E, Lyman GH. Predictors of reduced dose intensity in patients with early-stage breast cancer receiving adjuvant chemotherapy. Breast Cancer Res Treat. 2006;100(3):255-62. https://doi.org/10.1007/S10549006-9254-4.

18. Bleicher RJ, Ruth K, Sigurdson ER, et al. Time to surgery and breast cancer survival in the United States. JAMA Oncol. 2016;2(3):330-9. https://doi.org/10.1001/jamaoncol.2015.4508.

19. Eaglehouse YL, Georg MW, Shriver CD, Zhu K. Time-to-surgery and overall survival after breast cancer diagnosis in a universal health system. Breast Cancer Res Treat. 2019;178:441-50. https://doi.org/10.1007/s10549-019-05404-8.

20. Suleman K, Almalik O, Haque E, et al. Does the timing of surgery after neoadjuvant therapy in breast cancer patients affect the outcome? Oncology. 2020;98(3):168-73. https://doi.org/10.1159/ 000504964.

21. Omarini $\mathrm{C}$, Guaitoli $\mathrm{G}$, Noventa $\mathrm{S}$, et al. Impact of time to surgery after neoadjuvant chemotherapy in operable breast cancer patients. Eur J Surg Oncol. 2017;43(4):613-8. https://doi.org/10. 1016/j.ejso.2016.09.020.

22. Riba LA, Gruner RA, Fleishman A, James TA. Surgical risk factors for the delayed initiation of adjuvant chemotherapy in breast cancer. Ann Surg Oncol. 2018;25(7):1904-11. https://doi. org/10.1245/s10434-018-6351-7.

23. Yu K-D, Fan L, Qiu L-X, Ling H, Jiang Y-Z, Shao Z-M. Influence of delayed initiation of adjuvant chemotherapy on breast cancer survival is subtype-dependent. Oncotarget. 2017;8(28):46549-56. https://doi.org/10.18632/oncotarget. 10551.

24. Raphael MJ, Biagi JJ, Kong W, Mates M, Booth CM, Mackillop WJ. The relationship between time to initiation of adjuvant chemotherapy and survival in breast cancer: a systematic review and meta-analysis. Breast Cancer Res Treat. 2016;160(1):17-28. https://doi.org/10.1007/s10549-016-3960-3.

25. Abdel-Rahman O. Impact of timeliness of adjuvant chemotherapy and radiotherapy on the outcomes of breast cancer; a pooled analysis of three clinical trials. Breast. 2018;38:175-80. https://d oi.org/10.1016/j.breast.2018.01.010.

26. Kaufmann M, Von Minckwitz G, Mamounas EP, et al. Recommendations from an international consensus conference on the current status and future of neoadjuvant systemic therapy in 
primary breast cancer. Ann Surg Oncol. 2012;19(5):1508-16. h ttps://doi.org/10.1245/s10434-011-2108-2.

27. Akhtar Z, Stearns V, Cartwright P, et al. The effect of 1-day multidisciplinary clinic on breast cancer treatment. Breast Cancer Res Treat. 2020;182(3):623-9. https://doi.org/10.1007/s10549-0 20-05721-3.

28. Graham PJ, Brar MS, Foster T, et al. Neoadjuvant chemotherapy for breast cancer, is practice changing? A population-based review of current surgical trends. Ann Surg Oncol. 2015;22(10):3376-82. https://doi.org/10.1245/s10434-015-4714$\mathrm{x}$.

29. Ang E, Wewala N, Carroll R, et al. Neoadjuvant chemotherapy in non-metastatic breast cancer: a study on practice trends in a regional cancer treatment service. Intern Med J. 2020;50(3):315-21. https://doi.org/10.1111/imj.14326.

30. Eisen A, Hey A, Arnout A, Pinchuk B, Muradali D. Breast cancer pathway map I Cancer Care Ontario. Accessed 29 Jan 2021. h ttps://www.cancercareontario.ca/en/pathwaymap/breast-cancer-p athway-map.

31. NCCN. NCCN Clinical Practice Guidelines in Oncology (NCCN Guidelines $\left.{ }^{\circledR}\right)$ Breast Cancer. Version 12014. 2014.

32. Chia S, Swain SM, Byrd DR, Mankoff DA. Locally advanced and inflammatory breast cancer. J Clin Oncol. 2008;26(5):786-90. h ttps://doi.org/10.1200/JCO.2008.15.0243.

33. Mamtani A, Barrio AV, King TA, et al. How often does neoadjuvant chemotherapy avoid axillary dissection in patients with histologically confirmed nodal metastases? results of a prospective study. Ann Surg Oncol. 2016;23(11):3467-74. https://doi. org/10.1245/s10434-016-5246-8.
34. Rosenberg SM, Dominici LS, Gelber S, et al. Association of breast cancer surgery with quality of life and psychosocial wellbeing in young breast cancer survivors Supplemental content. JAMA Surg. 2020;155(11):1035-42. https://doi.org/10.1001/jama surg.2020.3325.

35. Sheng JY, Santa-Maria CA, Mangini N, et al. Management of breast cancer during the COVID-19 pandemic: a stage- and subtype-specific approach. JCO Oncol Pract. 2020. https://doi. org/10.1200/op.20.00364.

36. Fedewa SA, Edge SB, Stewart AK, Halpern MT, Marlow NM, Ward EM. Race and ethnicity are associated with delays in breast cancer treatment (2003-2006). J Health Care Poor Underserved. 2011;22(1):128-41. https://doi.org/10.1353/HPU.2011.0006.

37. Crown A, Muhsen S, Zabor EC, et al. Does use of neoadjuvant chemotherapy affect the decision to pursue fertility preservation options in young women with breast cancer? Oncology. 2020;27:4740-9. https://doi.org/10.1245/s10434-020-08883-y.

38. Wright F, De Vito C, Langer B. Multidisciplinary Cancer Conference Standards. Cancer Care Ontario. 2006. Accessed 30 Jan 2021. https://www.cancercareontario.ca/en/guidelines-advice/typ es-of-cancer/286.

39. Simmons CE, Hogeveen S, Leonard R, et al. A Canadian national expert consensus on neoadjuvant therapy for breast cancer: linking practice to evidence and beyond. Curr Oncol. 2015;22:2328. https://doi.org/10.3747/co.22.2328.

Publisher's Note Springer Nature remains neutral with regard to jurisdictional claims in published maps and institutional affiliations. 